\title{
Hydrometra Associated with Absence of the Vagina and Genital Tuberculosis.
}

\author{
By Daniel Dougal, M.C., M.D., Ch.B.(Victoria University), \\ Hon. Gynacological Surgeon, Northern Hospital; and Assistant \\ Surgeon, St. Mary's Hospitals, Manchester.
}

THF patient from whom I removed the specimen was a girl, aged I9 years. She was brought to me on account of delay in the onset of menstruation and because she suffered from extremely severe headaches occurring monthly and lasting three days. These headaches commenced twelve months previously. She also complained of always feeling tired and done up. Apart from the headaches, she had no actual pain.

The girl was well-developed and of healthy appearance, and had had no illnesses apart from scarlet fever and measles during childhood.

On abdominal examination, I found a mass above the pubes, rather to the left side, and apparently about the size of a foetal head. There was no tenderness. Inspection of the external genitals showed the vulva to be well-formed except that no vaginal orifice was present. There was a well-developed hymen of cribriform type, but underneath it merely a smooth skin surface. There was no bulging.

Rectal examination confirmed the absence of the vagina, and I was able to feel, high up in the pelvis, the tumour previously palpated through the abdominal wall.

I made a diagnosis of hæmatometra with absence of at least part of the vagina, and advised a careful examination under anæsthesia and an attempt to reach the retained blood from below. The mother was agreeable to this, and the patient was admitted to St. Mary's Hospital in due course. I incised the skin under the hymen and dissected up for about an inch between bowcl and bladder. Above this point these became very adherent, and as I was still at least two inches from the tumour, I thought it best to give up the attempt and explore later through the abdomen, after explaining the state of affairs to the patient's mother.

I opened the abdomen a week later and found the state of the pelvic organs to be as follows :-

The body of the uterus was somewhat enlarged, elastic in 


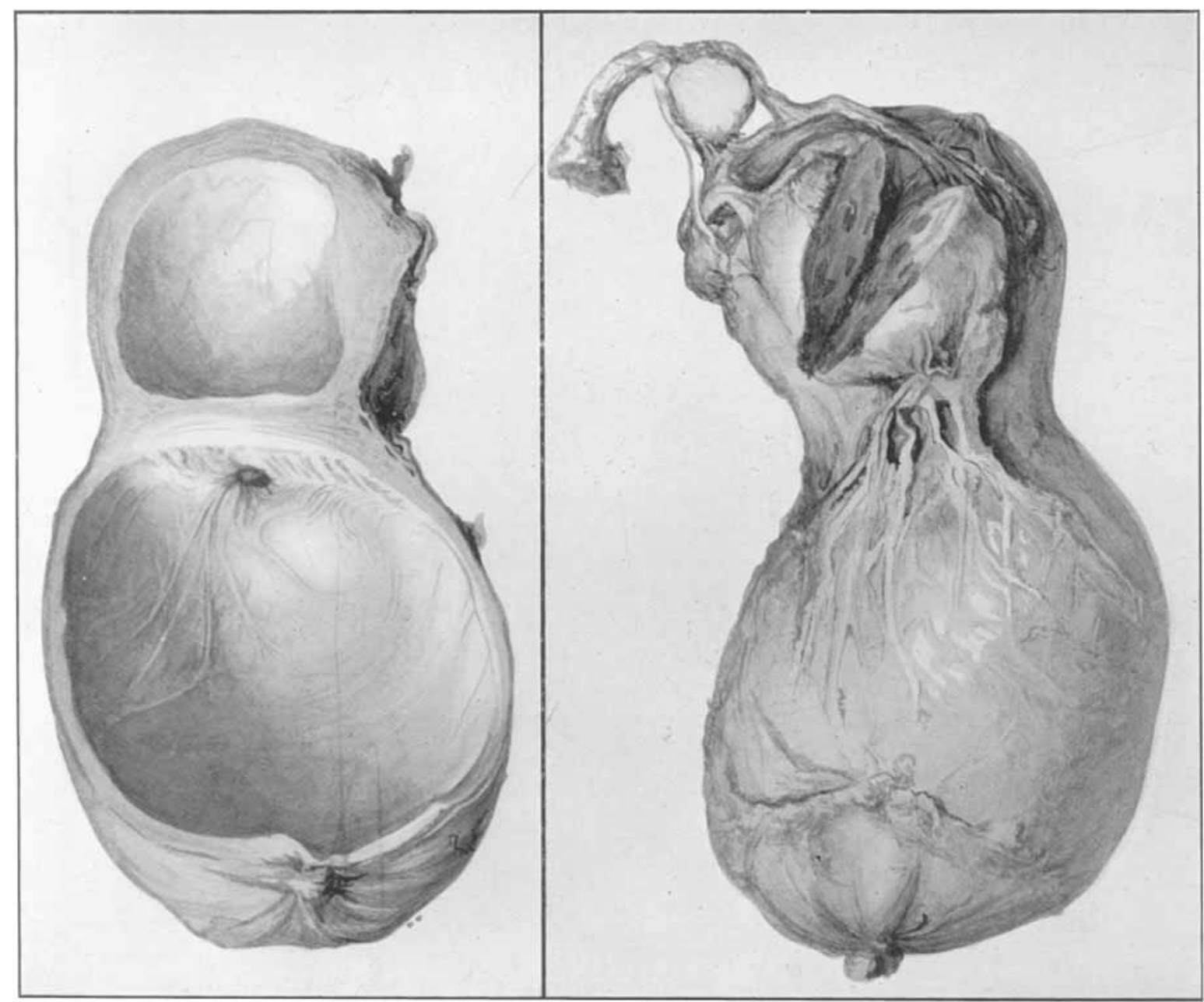

Dr. Dougal's case of Hydrometra associated with absence of the Vagina and Genital Tuberculosis. 
consistence and continuous below with a tense cystic swelling, the size of a goose's egg, which extended deep into the pelvis between the bladder in front and the rectum behind, the latter being adherent to its posterior surface.

There were numerous adhesions between the top of the uterus and the omentum, and near the right cornu was a yellow calcareous nodule the size of a bean.

The right Fallopian tube appeared to be absent, and was probably destroyed in the formation of scar-tissue round the nodule. The right ovary was cystic, and lay on the right wall of the pelvis to which it was adherent.

The left Fallopian tube and ovary were represented by an adherent mass fixed to the back of the broad ligament, and containing a quantity of calcareous material. Numerous calcareous glands were present at the ileo-cæeal junction.

I decided that any attempts at conservative measures would be useless, and proceeded to remove the uterus and appendages. After tying the infundibulo-pelvic ligaments on each side and dividing the broad ligaments, I commenced the separation of the lower cystic mass. The bladder was easily pushed off in front and the adherent bowel detached behind. The uterine vessels were small, and joined the lower cystic mass about the junction of the upper and middle thirds. On the left side the ureter was adherent to the mass just below the vessel, and had to be stripped off. The right ureter was well out of the way. After dividing two bands of tissue, which I took to be the utero-sacral ligaments, I was able to pull the whole mass up, and then found that it had a narrow attachment to the pelvic floor. On cutting this across some yellow mucinous material escaped from the tumour. The operation was completed in the usual way, and the patient made a good recovery.

The specimen is an interesting one, and consists of a dumb-bell shaped tumour, the upper part of which represents the body of the uterus and the lower part the cervix.

On cutting sagitally through the uterus two cavities are seen, both filled with thick tenacious mucus, containing large quantities of cholesterine crystals. The cavities communicate with each other by a circular opening one-third of an inch in diameter. The wall of the upper cavity is thick and muscular, but the lining membrane is flattened out and has a very incomplete covering of epithelium. No actively secreting cells or glands are visible. The lower cavity has a thinner and more fibrous wall, and the mucous membrane is well preserved, especially at the upper part. The epithelial lining consists of tall columnar cells, and there are many glands. The epithelial elements appear to be actually secreting mucus.

A section through the pedicle connecting the uterus with the 


\section{${ }_{63} 6$ Journal of Obstetrics and Gynæcology}

floor of the pelvis shows fibrous tissue only and no epithelial structures. Both ovaries contain follicles and corpora fibrosa.

In no part of the specimen could I find histological evidence of tubercle, but $I$ feel sure that a tuberculous peritonitis was present at some time in the patient's history, although $I$ have been unable to obtain any clinical evidence of it.

There are three definite lesions in this case:-

r. Total absence of the vagina.

2. Adnexal disease and peritoneal adhesions, presumably resulting from a tuberculous infection.

3. Distension of the cervix and body of the uterus with secretions as a result of excessive activity of the cervical mucous membrane and obstruction of the natural outlet.

Both ovaries were physiologically active, and, therefore, the absence of menstrual blood from the uterus must be explained by the fact that the hydrometra was present before puberty and either destroyed the mucous membrane of the body by pressure or raised the intra-uterine tension so high that bleeding could not occur.

In conclusion, I suggest that the sequence of events was as follows :- -

The absence of the vagina was developmental.

During infancy or childhood the patient had a tuberculous peritonitis with a resulting salpingo-öphoritis. In the course of this illness the uterus became extremely congested with a consequent increase in the amount of mucous secretions. The cervix, being larger than the body in the infantile uterus, would contribute a larger amount.

Having no natural outlet the secretions were retained and gradually distended the cervical canal and uterine cavity.

The patient reached puberty, no menstruation was possible on account of the hydrometra, but severe monthly headaches occurred, and led to her seeking advice. 